\title{
Facial Emotion Recognition Abilities in Women Suffering from Eating Disorders
}

\begin{abstract}
Wyssen, Andrea ; Junpeng, Lao ; Rodger, Helen ; Humbel, Nadine ; Lennertz, Julia ; Schuck, Kathrin ; Isenschmid, Bettina ; Milos, Gabriella ; Trier, Stephan ; Whinyates, Katherina ; Assion, Hans-Jörg ; Ueberberg, Bianca ; Müller, Judith ; Klauke, Benedikt ; Teismann, Tobias ; Margraf, Jürgen ; Juckel, Georg ; Kossmann, Christian ; Schneider, Silvia ; Caldara, Roberto ; Munsch, Simone
\end{abstract}

\begin{abstract}
OBJECTIVE Impairments in facial emotion recognition are an underlying factor of deficits in emotion regulation and interpersonal difficulties in mental disorders, and are evident in eating disorders (EDs). METHODS We used a computerized psychophysical paradigm to manipulate parametrically the quantity of signal in facial expressions of emotion (QUEST threshold seeking algorithm). This was used to measure emotion recognition in 311 adult women (anorexia nervosa $(\mathrm{AN}, \mathrm{n}=61)$, bulimia nervosa $(\mathrm{BN}, \mathrm{n}=58)$, healthy controls $(\mathrm{HC}, \mathrm{n}=130)$ and mixed mental disorders $($ mixed,n=59)). The mean age was 22.84 years $(\mathrm{SD}=3.90)$. The aim was to establish recognition thresholds defining how much information a person needs to recognize a facial emotion expression and to identify deficits in EDs compared to healthy and clinical controls. The stimuli included six basic emotion expressions (fear, anger, disgust, happiness, sadness, surprise) plus a neutral expression. RESULTS Happiness was discriminated at the lowest, fear at the highest threshold by all groups. There were no differences regarding thresholds between groups, except for the mixed and the BN group with respect to the expression of disgust $(\mathrm{F}(3,302)=5.97$, $\mathrm{p}=.001$, eta=.056). Emotional clarity, ED pathology and depressive symptoms did not predict performance (RChange .010, F(1,305) 5.74, p .079). The confusion matrix did not reveal specific biases in either group. CONCLUSIONS Overall, within-subject effects were as expected, whereas between-subject effects were marginal and psychopathology did not influence emotion recognition. Facial emotion recognition abilities in women suffering from EDs compared to women suffering from mixed mental disorders and HCs were similar. While basic facial emotion recognition processes seems to be intact, dysfunctional aspects such as misinterpretation might be important in emotion regulation problems. CLINICAL TRIAL REGISTRATION NUMBER DRKS-ID: DRKS00005709.
\end{abstract}

DOI: https://doi.org/10.1097/PSY.0000000000000664

Posted at the Zurich Open Repository and Archive, University of Zurich

ZORA URL: https://doi.org/10.5167/uzh-185532

Journal Article

Published Version

Originally published at:

Wyssen, Andrea; Junpeng, Lao; Rodger, Helen; Humbel, Nadine; Lennertz, Julia; Schuck, Kathrin; Isenschmid, Bettina; Milos, Gabriella; Trier, Stephan; Whinyates, Katherina; Assion, Hans-Jörg; Ueberberg, Bianca; Müller, Judith; Klauke, Benedikt; Teismann, Tobias; Margraf, Jürgen; Juckel, Georg; Kossmann, 
Christian; Schneider, Silvia; Caldara, Roberto; Munsch, Simone (2019). Facial Emotion Recognition Abilities in Women Suffering from Eating Disorders. Psychosomatic Medicine, 81(2):155-164. DOI: https://doi.org/10.1097/PSY.0000000000000664 


\title{
Facial Emotion Recognition Abilities in Women Experiencing Eating Disorders
}

\author{
Andrea Wyssen, PhD, Junpeng Lao, PhD, Helen Rodger, MSc, Nadine Humbel, MSc, Julia Lennertz, MSc, \\ Kathrin Schuck, PhD, Bettina Isenschmid, MD, Gabriella Milos, MD, Stephan Trier, MD, \\ Katherina Whinyates, MSc, Hans-Jörg Assion, MD, Bianca Ueberberg, MD, Judith Müller, MSc, \\ Benedikt Klauke, PhD, Tobias Teismann, PhD, Jürgen Margraf, PhD, Georg Juckel, MD, \\ Christian Kossmann, MD, Silvia Schneider, PhD, Roberto Caldara, PhD, and Simone Munsch, PhD
}

\begin{abstract}
Objective: Impairments in facial emotion recognition are an underlying factor of deficits in emotion regulation and interpersonal difficulties in mental disorders and are evident in eating disorders (EDs).

Methods: We used a computerized psychophysical paradigm to manipulate parametrically the quantity of signal in facial expressions of emotion (QUEST threshold seeking algorithm). This was used to measure emotion recognition in 308 adult women (anorexia nervosa $[n=61]$, bulimia nervosa $[n=58]$, healthy controls $[n=130]$, and mixed mental disorders [mixed, $n=59])$. The M (SD) age was 22.84 (3.90) years. The aims were to establish recognition thresholds defining how much information a person needs to recognize a facial emotion expression and to identify deficits in EDs compared with healthy and clinical controls. The stimuli included six basic emotion expressions (fear, anger, disgust, happiness, sadness, surprise), plus a neutral expression.

Results: Happiness was discriminated at the lowest, fear at the highest threshold by all groups. There were no differences regarding thresholds between groups, except for the mixed and the bulimia nervosa group with respect to the expression of disgust $\left(F(3,302)=5.97, p=.001, \eta^{2}=.056\right)$. Emotional clarity, ED pathology, and depressive symptoms did not predict performance $\left(R_{\text {Change }}^{2} \leq .010, F(1,305) \leq 5.74, p \geq .079\right)$. The confusion matrix did not reveal specific biases in either group.

Conclusions: Overall, within-subject effects were as expected, whereas between-subject effects were marginal and psychopathology did not influence emotion recognition. Facial emotion recognition abilities in women experiencing EDs compared with women experiencing mixed mental disorders and healthy controls were similar. Although basic facial emotion recognition processes seems to be intact, dysfunctional aspects such as misinterpretation might be important in emotion regulation problems.
\end{abstract}

Clinical Trial Registration Number: DRKS-ID: DRKS00005709.

Key words: anorexia nervosa, bulimia nervosa, eating disorders, facial emotion recognition, women.

\section{INTRODUCTION}

$\mathbf{R}^{2}$ ecognizing and decoding emotions in the faces of others is a core characteristic of human cognition and a fundamental feature of social interaction $(1,2)$. Previous research has shown that there are intra- and interindividual differences in observers' perceptual thresholds for the discrimination of facial emotion expressions. Happiness, surprise, fear, anger, disgust, and sadness are described as the six basic emotions, which cannot be broken down into smaller segments (see e.g., (3)). Among these emotions, happiness can be discriminated at the lowest threshold. Conversely, much more information is needed to correctly identify fear (4). A recent study identified that the average recognition threshold for fear is $97 \%$ signal strength, whereas it is only an average of $35 \%$ for happiness (5). In addition, there is evidence underlining a large intraindividual variability in emotion recognition in adults, i.e., in terms of signal strength to correctly recognize an emotion expression (e.g., (5)).

AN = anorexia nervosa, ANCOVA = analysis of covariance BDI-II = Beck Depression Inventory, BED = binge-eating disorder, $\mathbf{B M I}=$ body mass index, $\mathbf{B N}=$ bulimia nervosa, $\mathbf{D E R S}=$ Difficulties with Emotion Regulation Scale, DIPS = Diagnostisches Interview für Psychische Störungen, ED = eating disorder, EDE-Q = Eating Disorder Examination-Questionnaire, $\mathbf{H C}=$ healthy control, $\mathbf{R M E T}=$ Reading the Mind in the Eye Task

\section{SDC Supplemental Content}

From the Departments of Clinical Psychology and Psychotherapy (Wyssen, Humbel, Munsch) and Visual and Social Neuroscience (Lao, Rodger, Caldara), University of Fribourg, Fribourg, Switzerland; Faculty of Psychology, Mental Health Research and Treatment Center (Lennertz, Schuck, Teismann, Margraf, Schneider), Ruhr University Bochum, Bochum, Germany; Kompetenzzentrum für Essverhalten (Isenschmid), Adipositas und Psyche Spital Zofingen; Departement of Consulation-Liaison Psychiatry and Psychosomatic Medicine (Milos), University Clinic Zürich, Zürich, Switzerland; Privatklinik Aadorf (Trier), Aadorf; Klinik Schützen (Whinyates), Rheinfelden, Switzerland; Department of Psychiatry, Psychotherapy and Psychosomatic Medicine (Assion, Ueberberg), LWL-Klinik Dortmund; Department of Psychiatry, Psychotherapy, Psychosomatic and Preventive Medicine (Juckel, Kossmann), LWL-Klinik Bochum, Ruhr University Bochum, Bochum, Germany; and Christoph-Dornier-Clinic for Psychotherapy (Müller, Klauke), Münster, Germany. Address correspondence to Simone Munsch, PhD, Institute of Psychology, Department of Clinical Psychology and Psychotherapy, University of Fribourg, Rue de Faucigny 2, 1700 Fribourg, Switzerland. E-mail: simone.munsch@unifr.ch

Caldara and Munsch shared last authorship.

Received for publication January 17, 2018; revision received October 31, 2018.

DOI: 10.1097/PSY.0000000000000664

Copyright (C) 2019 by the American Psychosomatic Society 
Kret and Ploeger (6) proposed disrupted processing of emotion expression in the faces of others as an important factor underlying mental disorders, which could partly explain high comorbidity rates. They envisaged two main steps of emotion processing: first, emotion perception, described as the attention to emotional stimuli in the environment, and the correct identification and adequate response to the emotion. Second, emotion regulation defined as managing one's own emotional state. To date, there is considerable evidence for the second step of the model, e.g., increased levels of suppression or rumination in depressive or anxiety disorders (e.g., $(7,8))$. However, our understanding of similarities and disorder-specific deficits related to the first part of the model (i.e., basic mechanisms as the processes underlying emotion recognition) is still incomplete. In the present study, we assess emotion recognition via recognition thresholds as well as via confusion rates. The basic assumption (following established theories, see e.g. (6)) is that recognition thresholds and confusion rates represent aspects of emotion recognition accuracy. The recognition threshold is more related to impaired interpretation of emotional facial stimuli, whereas the confusion rate is related to an attentional bias (e.g., a bias toward negative emotions).

There is growing evidence on impairments in facial emotion recognition irrespective of task type in patients with borderline personality disorder $(9,10)$, schizophrenia (11), autism spectrum disorders (12), bipolar disorder (13), major depressive disorder (14), and posttraumatic stress disorder (15). Impairment in emotion recognition in these groups of patients is pronounced and often includes a biased perception such as a heightened sensitivity to negative emotions, (16) e.g., to fear and disgust in anxiety disorders $(15,17)$, influences subsequent processes such as the interpretation of a situation, and thus is part of an overall problematic social cognitive functioning. Eating disorders (EDs) have also been linked to emotion processing deficits $(6,18)$ as well as to problems in social cognition and social interactions (19). In their "cognitive-interpersonal maintenance model of anorexia nervosa," Treasure and Schmidt (18) describe an automatic attentional bias toward negative emotions and an impaired interpretation of emotions related to problems in social interaction and social anhedonia in anorexia nervosa (AN). Difficulties in social communication and the avoidance of close relationships are important features AN because they foster ED characteristic behaviors and beliefs. This leads to social isolation, which is associated with less social support, and fewer social activities, which connects to other common characteristics of patients with EDs such as inhibition, shyness, and a tendency to internalize problems (18).

Recent studies investigating emotion recognition abilities in EDs often applied the Reading the Mind in the Eye Task (RMET) (20). For each of the 36 gray-scale cropped photos of eyes, the most appropriate word among four mental state terms is selected as the probable correct description of the person feelings whose eyes are shown (21). The RMET represents not only an elementary emotion recognition test but also a theory of mind test because it is necessary to project one's thoughts into the mind of the person in the picture to attribute the correct mental state (22). For ED patients, results on the RMET were ambiguous: some found reduced emotion recognition in ED patients, especially in individuals with AN (23-25), whereas results for patients with $\mathrm{BN}$ are less clear. Several authors report similar performances for BN patients and HCs (25-27), whereas others referred to difficulties, e.g. in reading positive emotions and neutral states (28). According to the critique that responses of the RMET are simply coded as right or wrong, more subtle tasks have been developed, where emotion recognition is assessed by computerized distorted facial pictures such as morphing or variations in signal strength (29). A small group of patients with $\mathrm{AN}(n=28)$ was less accurate in identifying basic emotions than HCs $(n=28)$ when applying a facial emotion recognition task with the six basic emotions, which were displayed using morphs (from neutral to the full expression) (30). In contrast, no difference in emotion recognition was found between $\mathrm{HCs}(n=73)$ and patients with $\mathrm{EDs}(n=49 \mathrm{AN}, n=16 \mathrm{BN})$ when participants rated video clips displaying a discrete emotion (happy, sad, anger, neutral) (31). Using pictures of faces portraying emotions with varying levels of ambiguity, less accurate recognition of disgust and an error response bias toward anger in AN patients $(n=35)$ compared with HCs $(n=42)$ was found (32). There is further evidence for reduced accuracy in recognizing subtle emotional expressions in nonclinical participants with ED symptoms $(n=40)$ compared with HCs $(n=40)$ in a facial emotion recognition test with static images of faces each representing one of five emotions (happiness, sadness, anger, fear, and disgust) on different intensity levels (33). In addition, two studies investigated attention processes influencing emotion recognition in terms of reaction times in patients with $\mathrm{AN}$ and $\mathrm{BN}$. They found a bias toward rejecting versus accepting faces and difficulties to shift attention away from rejection faces especially in individuals with a current or history of EDs compared with HCs in a dot-probe task (34). Moreover, AN patients revealed an attention bias toward angry faces and a reduced capacity in processing of positive facial emotion expressions compared with HCs and obese individuals (35). This was in an experimental task in which adjectives had to be rated regarding their positive or negative value after a short priming with a schematic facial emotion expression (happy, sad, angry, neutral). However, recent studies in samples experiencing other mental disorders or HCs have revealed rather limited psychometric values of the dot-probe paradigm in terms of internal consistency or test-retest reliability (36).

Brewer and colleagues (37) applied a novel facial emotion recognition task, in which they showed basic facial emotion expressions (happiness, sadness, surprise, fear, disgust, anger, and pain). They used gray-scaled images with different levels of visual noise for 800 milliseconds in an adaptive stair case procedure to determine the maximal level of noise at which an individual could still recognize an emotion reliably. They found that in 21 women with EDs (AN and $\mathrm{BN}$ ) and 21 healthy women, the level of co-occurring alexithymia was predictive of poorer emotion recognition ability, whereas the degree of ED pathology was not. Lule and colleagues (38) reported that in a sample of 15 female adolescents with $\mathrm{AN}$ and 15 matched HCs using the facial expressed emotion labeling test (six basic emotions: anger, fear, sadness, surprise, disgust, and happiness, presented for 300 milliseconds), patients showed impairments in correctly identifying the emotion disgust, whereas they performed better in the recognition of happiness. However, after controlling for depression, group differences disappeared. Current evidence (32) refers to a possible influence of body mass index (BMI) and length of illness in AN patients $(n=35)$ on recognition of disgust using pictures depicting blended emotions with different levels of ambiguity. No effects have been found for depression, obsessive-compulsive symptoms, alexithymia, and psychotropic medication (32). 
To sum up, although there are indications of more pronounced difficulties in, e.g., the RMET in AN, findings in $\mathrm{BN}$ are less clear (25-27). Applying more subtle methods assessing emotion recognition by computerized distorted facial pictures such as morphing or variations in signal strength or focusing on attentional patterns did not reveal a clear line of results. Although some studies found differences between EDs and healthy participants, others did not (29). Additional findings of previous studies in samples of AN and $\mathrm{BN}$ patients compared with HCs showed that comorbid depression, duration of illness, BMI, and alexithymia are associated with poorer emotion recognition performance.

This lack of consistent knowledge regarding the ability to identify correctly emotions is detrimental, influences social cognition, and plays an important role in the "cognitive-interpersonal maintenance model of anorexia nervosa" (18). Disturbed eating behavior (e.g., restriction of food intake, binge eating, and vomiting) is thought to be triggered by deficient emotion regulation after social conflicts $(18,25,39)$. A training of emotion processing, including emotion recognition, could contribute to an efficacious treatment because it addresses highly relevant maintaining factors (40).

The objectives of our study were therefore twofold: first, we aimed to assess individual facial emotion recognition thresholds in a large sample of women with $\mathrm{AN}$ and $\mathrm{BN}$ and compare them with a group of women with mixed mental disorders (depressive and anxiety disorders) and healthy women. Based on preliminary findings (30,32), we assumed impairments in terms of a higher signal threshold in emotion recognition in $\mathrm{AN}$, but not in $\mathrm{BN}$ patients, where we expected similar performance as in HCs. The mixed control group of patients experiencing depressive and anxiety disorder was included to explore the question of similarity or specificity of emotion recognition deficits. Second, response profiles for each facial expression, including their confusion with other expressions, were analyzed to identify biases for certain emotions. Based on existing evidence $(28,34,35)$, we attempted to validate previous findings of a more pronounced biases toward negative/ rejecting facial emotion expressions (such as angry faces) in patients experiencing $\mathrm{AN}$ and $\mathrm{BN}$.

In our analyses, we controlled for age and examined the role of potential patient-related factors such as BMI, emotional clarity (i.e., the extent to which an individual understands the emotions that it is experiencing), depressiveness, and degree of ED pathology. Whereas many previous studies have used the RMET or prototypical pictures of faces that show an emotion expression very intensely, we favored the novel psychophysical method QUEST, an adaptive staircase procedure (originally developed by (41)) to measure recognition thresholds for expression discrimination in a signal detection paradigm. The sensitivity of this procedure allows the detection of subtle emotion recognition difficulties, which may nevertheless impair social functioning and contribute to the maintenance of ED pathologies (33). It is the first time that the QUEST paradigm has been used in a large sample of patients experiencing $\mathrm{AN}, \mathrm{BN}$, and mixed mental disorders and compared with an $\mathrm{HC}$ group.

\section{METHOD}

\section{Participants}

Altogether 381 adult women from the age of 18 to 35 years were eligible to participate in the study. The assessment of emotion recognition capacities was part of a large cross-sectional and longitudinal multicenter study (42) investigating moderators of the effect of media on body image in women experiencing EDs versus experiencing mixed mental disorders and HCs. Data were collected between November 2012 and February 2017.

An inclusion criterion for all participants was age between 18 and 35 years. In total, 67 participants dropped out during the assessment period before the application of the emotion recognition task (26 HC, 6 AN, 13 $\mathrm{BN}, 22$ mixed). Another three participants $(2 \mathrm{AN}, 1 \mathrm{HC})$ were excluded from the analyses because of only providing "don't know" response during the task. Of the remaining participants, 130 were healthy female students who did not meet current or former criteria for any mental disorder according to the DSM-IV-TR (43). The three groups of patients included 61 women with AN, 58 with BN, and 59 women experiencing either from a depressive $(n=36)$ or from an anxiety disorder $(n=23)$. Comorbidity rates in our ED samples were comparable with the literature $(44,45)$, approximately $50 \%$ revealing one or more comorbid diagnosis. A total of $36.1 \%$ of AN patients experienced a comorbid depressive disorder, $19.7 \%$ from a comorbid anxiety disorder, whereas $43.1 \%$ of BN patients presented a comorbid depressive disorder and $27.6 \%$ with a comorbid anxiety disorder. For the mixed sample, the presence of an ED was an exclusion criterion. All mental disorders were assessed according to the DSM-IV-TR criteria. In the patients' group, 59\% were treated in an inpatient and $41 \%$ in an outpatient setting. Exclusion criteria for study participation were psychotic disorders and pregnancy as well as other mental or physical conditions that would not have allowed the participation in the laboratory experiment or the inability to give informed consent. Healthy participants were bachelor or master students at the University of Fribourg (Switzerland) and had at least secondary school level. Patients were recruited from different psychiatric units in Switzerland and from psychiatric and psychotherapeutic units in Germany. Forty-seven percent of all participants were Swiss, $47 \%$ were German, and $6 \%$ belonged to another nationality. Participants were compensated for their time with course credits (students) or money (patients). The study protocol was approved by the local human ethics committees of the Department of Psychology of the University of Fribourg (Protocol Number 2012_001), the canton of Fribourg (Protocol Number 023/12-CER-FR), Aargau (Protocol Number 2013/057), Zürich (Protocol Number 2013-0457), and Thurgau (Protocol Number 2013/ 24), as well as by the local ethics committees of the Department of Psychology at the Faculty of Medicine of the Ruhr University Bochum (Protocol Number 142). Informed consent was obtained from all participants. Table 1 displays characteristics of the present samples.

\section{Instruments and Materials}

In the following, measures included in this substudy are introduced. For an introduction to the overall assessment, refer to Munsch (42).

\section{Diagnostic Interview}

The Diagnostisches Interview für psychische Störungen (DIPS) (46) is a structured diagnostic interview based on the DSM-IV-TR (43) with good reliability and validity. Ten percent of the interviews in the present study were coded twice. Interrater reliability was satisfying with $.80, .85$ for primary diagnosis, and .90 for comorbidity (Fleiss $\mathrm{k}$ ). The interview was conducted in a face-to-face inpatient settings. For all other participants, the DIPS was applied via telephone. The validity of telephone-based interviews is comparable to face-to-face interviews when screening for diagnoses (47). The interviews were conducted by trained and supervised master students in clinical psychology or postgraduate psychologists.

\section{Body Mass Index}

Weight and height were measured at the end of the experiment (for a detailed description see (42)) on an electronic balance scale (Seca) and by a stadiometer (Seca) with participants wearing clothing without shoes. BMI was calculated as weight in kilograms divided by the square of height in meters. 
TABLE 1. Sample Characteristics $(N=308)$

\begin{tabular}{|c|c|c|c|c|c|c|c|}
\hline & \multirow{2}{*}{$\frac{\mathrm{HC}(n=130)}{\mathrm{M}(\mathrm{SD})}$} & \multirow{2}{*}{$\frac{\mathrm{AN}(n=61)}{\mathrm{M}(\mathrm{SD})}$} & \multirow{2}{*}{$\begin{array}{c}\mathrm{BN}(n=58) \\
\mathrm{M}(\mathrm{SD})\end{array}$} & \multirow{2}{*}{$\frac{\text { Mixed }(n=59)}{M(S D)}$} & \multicolumn{2}{|c|}{ Statistics ${ }^{a}$} & \multirow[b]{2}{*}{ Bonferroni Post hoc ${ }^{b}$} \\
\hline & & & & & $F$ & $p$ & \\
\hline Age & $21.53(2.18)$ & $22.87(4.57)$ & $23.16(3.96)$ & $25.29(4.79)$ & 11.46 & $* * *$ & Mixed $>\mathrm{HC}^{* * *}, \mathrm{AN}^{* *}, \mathrm{BN}^{* *} ; \mathrm{HC}<\mathrm{AN}^{*}, \mathrm{BN}^{* *}$ \\
\hline BMI & $22.01(2.63)$ & $17.05(1.63)$ & $22.64(2.56)$ & $24.32(6.45)$ & 39.61 & $* * *$ & $\mathrm{AN}<\mathrm{HC}, \mathrm{BN}$, mixed $^{* * *}$, mixed $>\mathrm{HC}^{* * *}, \mathrm{BN}^{*}$ \\
\hline BDI-II & $3.89(4.71)$ & $24.50(11.15)$ & $25.78(11.63)$ & $25.48(9.16)$ & 117.85 & $* * *$ & $\mathrm{HC}<\mathrm{AN}, \mathrm{BN}$, mixed $^{* * *}$ \\
\hline DERS total & $67.32(16.68)$ & $110.73(24.13)$ & $114.19(24.30)$ & $113.94(23.32)$ & 107.06 & $* * *$ & $\mathrm{HC}<\mathrm{AN}, \mathrm{BN}$, mixed $^{* * *}$ \\
\hline DERS clarity & $8.72(2.62)$ & $16.07(4.91)$ & $16.43(5.29)$ & $15.33(4.55)$ & 62.67 & $* * *$ & $\mathrm{HC}<\mathrm{AN}, \mathrm{BN}$, mixed $^{* * *}$ \\
\hline EDE-Q & $0.76(0.70)$ & $3.53(1.23)$ & $4.06(1.31)$ & $1.50(1.28)$ & 142.59 & $* * *$ & $\begin{array}{l}\mathrm{HC}<\mathrm{AN}, \mathrm{BN}, \text { mixed }{ }^{* * *} ; \mathrm{AN}<\mathrm{BN}^{*} ; \\
\text { mixed }<\mathrm{AN}, \mathrm{BN}^{* * *} ; \mathrm{HC}<\text { mixed }^{* * *}\end{array}$ \\
\hline
\end{tabular}

$\mathrm{HC}=$ healthy controls; $\mathrm{AN}=$ anorexia nervosa $\mathrm{BN}=$ bulimia nervosa; Mixed = mixed mental disorders; BMI = body mass index; $\mathrm{BDI}-\mathrm{II}=\mathrm{Beck}$ Depression Inventory; EDE-Q = Eating Disorder Examination-Questionnaire; DERS = Difficulties in Emotion Regulation Scale.

$* p<.05$.

$* * p<.01$.

$* * * p<.001$.

${ }^{a}$ One-way ANOVA between diagnostic groups, $d f$ age $(3,190.86), d f$ BMI $(3,100.72), d f$ BDI-II $(3,182.77), d f$ DERS total $(3,212.10), d f$ DERS awareness $(3,232.34), d f$ DERS clarity $(3,192.75)$, and $d f$ EDE-Q $(3,197.27)$; because analyses showed a violation of the assumption of homogeneity of variances, the results of the Brown-Forsythe test, which provides good robustness, are reported.

${ }^{b}$ Bonferroni post hoc test, only significant relationships are reported.

\section{Questionnaires}

Standardized questionnaires in German were presented via an online survey platform (LimeSurvey).

\section{Depressive Symptoms (BDI-II)}

The "Beck Depression Inventory" (BDI-II) was used to assess depressive symptoms. It consists of 21 items and measures the severity of depressive symptoms during the previous 2 weeks (48). The instrument has good validity and reliability $(48,49)$. Cronbach's $\alpha$ in this sample was .96 .

\section{Eating Disorder Pathology}

The "Eating Disorder Examination-Questionnaire" (EDE-Q; German Version by (50)), is a self-report version of the Eating Disorder Examination (EDE (51)) assessing ED behaviors and symptoms during the past 28 days. It consists of 28 items; 22 items address attitudinal aspects of ED pathology. These items are rated on a scale from 0 to 6 and can be summarized into a global score as well as the following four subscales: restraint eating, eating concern, shape concern, and weight concern. Six additional items assess the frequency of key ED behaviors. The EDE-Q has good psychometric properties and is suitable to detect symptoms of EDs in individuals of the general population (52). In the present sample, the values were between .90 and .97 .

\section{Emotion Regulation}

The "Difficulties with Emotion Regulation Scale" (DERS (53)) (German version by (54)) is a 36-item self-report questionnaire assessing six dimensions of emotion regulation (none acceptance of emotions, difficulties in engaging in goal directed behavior, impulse control difficulties, lack of emotional awareness, limited access to emotion regulation strategies, and lack of emotional clarity) on a scale from 1 to 5 . All scales showed good internal consistencies and validity $(53,54)$. Cronbach's $\alpha$ of the DERS global score in the present sample was .97 .

\section{Emotion Recognition}

The QUEST threshold seeking algorithm was implemented in MATLAB 7.10.0. QUEST is a Bayesian adaptive staircase procedure for establishing an observer's threshold sensitivity to some physical attributes of a stimulus (41), providing a measure of how effectively an observer can discriminate a stimulus. Here, we investigated threshold sensitivity for signal of facial expression of emotions across groups. For more information see Text S1 and Figure S1, Supplemental Digital Content, http://links.lww.com/ PSYMED/A531.

\section{Procedure}

This work is part of a multicenter study (for a detailed description of the overall study protocol, see (42)).

Participants were introduced to the computerized emotion recognition task and told that they would see a series of facial expressions displaying a specific emotion. They were asked to indicate as accurately as possible which emotional expression they recognized by pressing the corresponding key on a keyboard. The participants were also told that the response time was not important and that they could take as much time as needed to give their response. The facial expression would be shown only briefly (500 milliseconds). Notice that the 500-millisecond presentation time was chosen because it allows sufficient time for emotion recognition to occur according to previous studies $(55,56)$ and we do not expect an effect of presentation time. Event-related potential studies show that differences among emotional expressions are processed from 140 milliseconds (55). The experiment took approximately 10 to 15 minutes to complete. For more information, see Text S2, Supplemental Digital Content, http://links.lww.com/PSYMED/A531.

\section{Data Analyses}

\section{Threshold Detection}

The standard implementation of the QUEST procedure is provided in the Psychtoolbox Version $3((41))$ as a toolbox in a subdirectory. It contains a collection of functions that extracts different quantity related to the threshold from the psychometric curved. Here, we use one of the MATLAB function (QuestMean.m) from the toolbox to compute the mean of the QUEST posterior probability density function (pdf) as the threshold for each participant. For more information, see Text S3, Supplemental Digital Content, http://links.lww.com/PSYMED/A531.

\section{Threshold Differences Across Groups}

To test whether emotion recognition thresholds were different in the four groups, a repeated measures analysis of covariance (ANCOVA) was performed with the within-subject factor emotion and the between-subject 
factor group $(7 \times 4)$; age was entered as covariate. We computed multiple regression models to qualify whether the expected differences in emotion recognition was the result of the intervening variables (BMI, depressiveness, ED pathology, and emotional clarity). Therefore, we predicted emotion recognition threshold with age (step 1) and the respective intervening variable (step 2). This analysis was performed for each emotion separately.

\section{Confusion Matrix Analysis}

Although the QUEST procedure is efficient in estimating the desired threshold, misclassification from the participant is not well represented in a multinomial setting such as the current experiment. Thus, to explore fully the individual response profile during each task, we also analyzed the confusion matrix of participant's response regardless of the presented stimuli signal level. For each participant, we constructed a confusion matrix where each row displays one of the six presented facial expressions plus neutral, and each column represents the response given by the observers. The values in the matrix thus show the frequency of response, conditioned on the presented stimulus. Ideally, the diagonal of the matrix, which represents the correct identification, should be at $75 \%$ by design of the task. However, this is not necessarily the case because some expressions could not be correctly identified at $75 \%$ even at the maximum signal level and we applied a stopping rule. Moreover, we did not consider Don't Know response as confusion, and therefore, the sum of each row could be less than $100 \%$. Nonetheless, the resulting matrix quantifies the common confusion made by the participant during the task. We then computed the average confusion matrix for each group to compare the response profile.

Moreover, to explore the (dis)similarity between and within each group, we computed full representational similarity matrix (57) by calculating the Pearson correlation of the confusion matrix between each unique pair of participants. Similarities within and between the groups are compared using a bootstrapped $95 \%$ confidence interval.

\section{RESULTS}

\section{Sample Characteristics}

Participant's characteristics are presented in Table 1. The mixed group was significantly older than the other groups. As expected, $\mathrm{AN}$ and $\mathrm{BN}$ patients had higher ED pathology values than the
$\mathrm{HC}$ group and the mixed group. The $\mathrm{HC}$ group significantly differed from the patients groups on depressive symptoms (BDI-II) and emotion regulation (DERS). The AN group showed significantly lower values on BMI than all other groups.

\section{Facial Emotion Expression Recognition Thresholds Across Groups}

The repeated measures ANCOVA (covariate age) revealed significant between- and within-subject effects (dependent variable recognition threshold): $F(3,302)=3.15, p=.025, \eta^{2}=.030$, $F(4.65,1404.66)=21.89, p<.001, \eta^{2}=.068$. The interaction between the within-subject factor (emotion) and the covariate (age) was not significant $\left(F(4.65,1404.66) \leq 1.12, p=.35, \eta^{2}=.004\right)$. The interaction group by emotion $(F(13.95,1404.66)=1.83$, $\left.p=.030, \eta^{2}=.018\right)$ was significant. Huynh-Feldt's correction was applied to the within-subject factor, because Mauchly's test of sphericity was significant. As displayed in Figure 1, all groups performed best for the emotion happiness, which had the lowest perceptual threshold.

The one way ANCOVA (controlling for age) revealed no significant effect of the between-subject factor group on the recognition of the emotion happiness $\left(F(3,302)=0.32, p=.814, \eta^{2}=.003\right)$, surprise $\left(F(3,302)=0.11, p=.952, \eta^{2}=.001\right)$, fear $(F(3,301)=1.42$, $\left.p=.238, \eta^{2}=.014\right)$, sadness $(F(3,302)=0.85, p=.465$, $\left.\eta^{2}=.008\right)$, anger $\left(F(3,302)=1.56, p=.198, \eta^{2}=.015\right)$, and neutral $\left(F(3,302)=1.51, p=.212, \eta^{2}=.015\right)$. A significant effect was found for the emotion disgust $(F(3,302)=5.97, p=.001$, $\eta^{2}=.056$ ). Contrast analyses (contrast 1 : HC versus AN; contrast 2: $\mathrm{HC}$ versus $\mathrm{BN}$; contrast 3: $\mathrm{HC}$ versus mixed; contrast 4: AN versus $\mathrm{BN}$; contrast 5: AN versus mixed; contrast 6: BN versus mixed) showed that the mixed group needed significantly more information to correctly identify disgust than the HC group, $t(85.51)=-3.12$, $p=.002, d=0.49$, and the AN group, $t(106.28)=-3.26$, $p=.002, d=0.59$. Moreover, the $\mathrm{BN}$ group performed worse than the HCs, $t(89.76)=-2.20, p=.030, d=0.35$, and the AN group, $t(109.66)=-2.43, p=.017, d=0.40$. Bonferroni correction was applied because of alpha error accumulation with multiple testing.

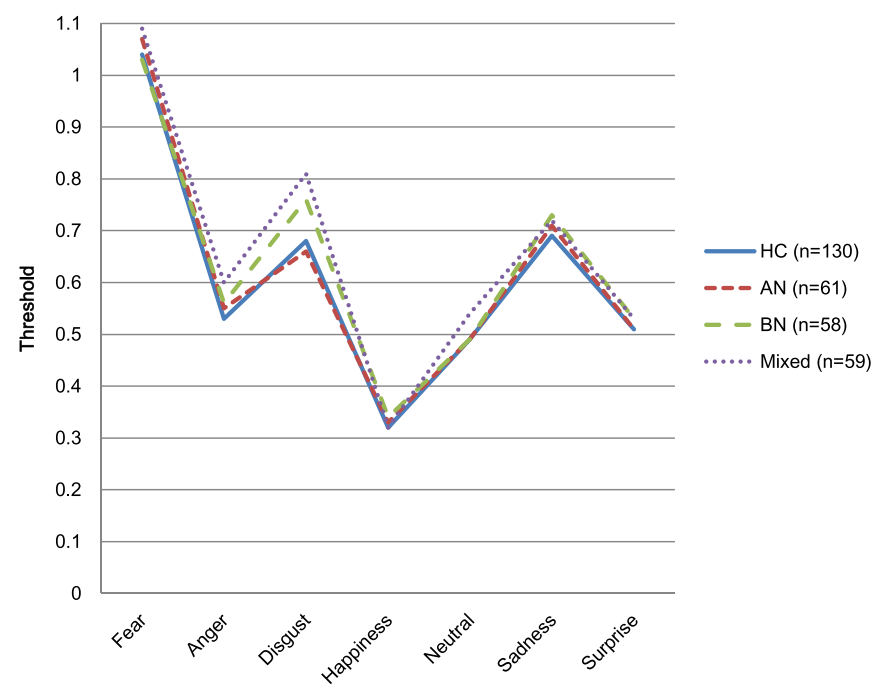

FIGURE 1. Mean emotion recognition thresholds across groups $(N=308)$. Notes: Mean values of thresholds were reported. Covariates in the model were calculated with age $=22.82$. Color image is available only in online version (www.psychosomaticmedicine.org). 
TABLE 2. Mean Emotion Recognition Thresholds (Signal Strength Where Expression Is Predicted to be Discriminated on $75 \%$ of Trials) Across Groups $(N=308)$

\begin{tabular}{|c|c|c|c|c|}
\hline & $\mathrm{HC}(n=130)$ & AN $(n=61)$ & $\mathrm{BN}(n=58)$ & Mixed $(n=59)$ \\
\hline Threshold & $M(S D)$ & $M(S D)$ & $M(S D)$ & $M(S D)$ \\
\hline Fear & $1.04(0.23)$ & $1.07(0.21)$ & $1.03(0.24)$ & $1.09(0.18)$ \\
\hline Anger & $0.53(0.13)$ & $0.55(0.13)$ & $0.56(0.13)$ & $0.60(0.19)$ \\
\hline Disgust & $0.68(0.20)$ & $0.66(0.20)$ & $0.76(0.24)$ & $0.81(0.28)$ \\
\hline Happiness & $0.32(0.10)$ & $0.33(0.04)$ & $0.34(0.07)$ & $0.32(0.10)$ \\
\hline Neutral & $0.49(0.13)$ & $0.49(0.11)$ & $0.49(0.11)$ & $0.54(0.15)$ \\
\hline Sadness & $0.69(0.20)$ & $0.71(0.20)$ & $0.73(0.21)$ & $0.72(0.20)$ \\
\hline Surprise & $0.51(0.16)$ & $0.51(0.16)$ & $0.53(0.16)$ & $0.53(0.16)$ \\
\hline
\end{tabular}

$\mathrm{HC}=$ healthy controls $; \mathrm{AN}=$ anorexia nervosa $; \mathrm{BN}=$ bulimia nervosa $;$ mixed $=$ mixed mental disorders. Lower threshold indicate more effective discrimination.

Lower threshold indicate more effective discrimination.
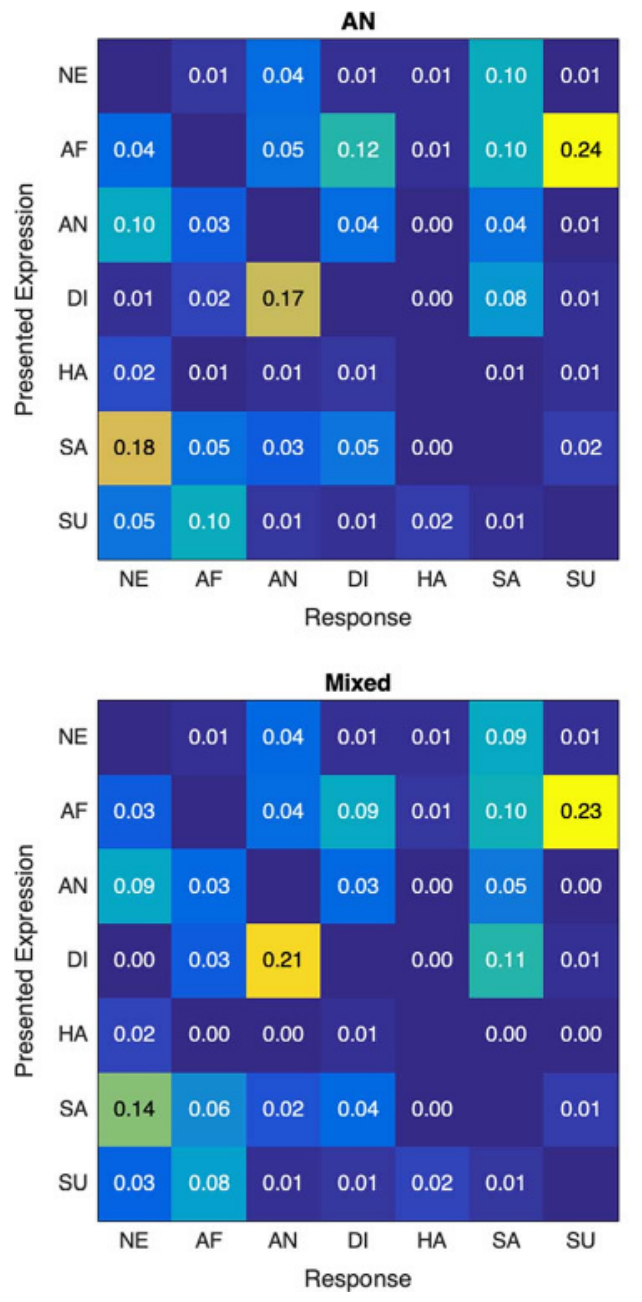

The mean values and standard deviation for each emotion expression divided into the diagnostic groups were presented in Table 2.

\section{The Influence of Depressiveness and ED Pathology on Emotion Recognition Thresholds}

Statistical tests with multiple linear regression analyses showed that age has a small but significant effect on the recognition threshold of the emotion anger $\left(R_{\text {Change }}^{2}=.027, F(1,305)=8.45\right.$, $p=.004)$ and surprise $\left(R_{\text {Change }}^{2}=.015, F(1,305)=4.55\right.$, $p=.034)$. None of the intervening variables (BMI, emotional clarity, depressiveness, ED pathology) explained additional variance in either of the emotion recognition thresholds: fear $\left(R_{\text {Change }}^{2} \leq .004, F(1,305) \leq 0.62, p \geq .269\right)$, anger $\left(R_{\text {Change }}^{2} \leq .006, F(1,305) \leq 5.74, p \geq .085\right)$, disgust $\left(R_{\text {Change }}^{2} \leq .010, F(1,305) \leq 1.98, p \geq .079\right)$, happiness $\left(R_{\text {Change }}^{2} \leq .002, F(1,305) \leq 0.94, p \geq .366\right)$, sadness $\left(R_{\text {Change }}^{2} \leq .005, F(1,305) \leq 0.82, p \geq .202\right)$, and surprise $\left(R_{\text {Change }}^{2} \leq .008, F(1,305) \leq 3.45, p \geq .112\right)$. The only significant
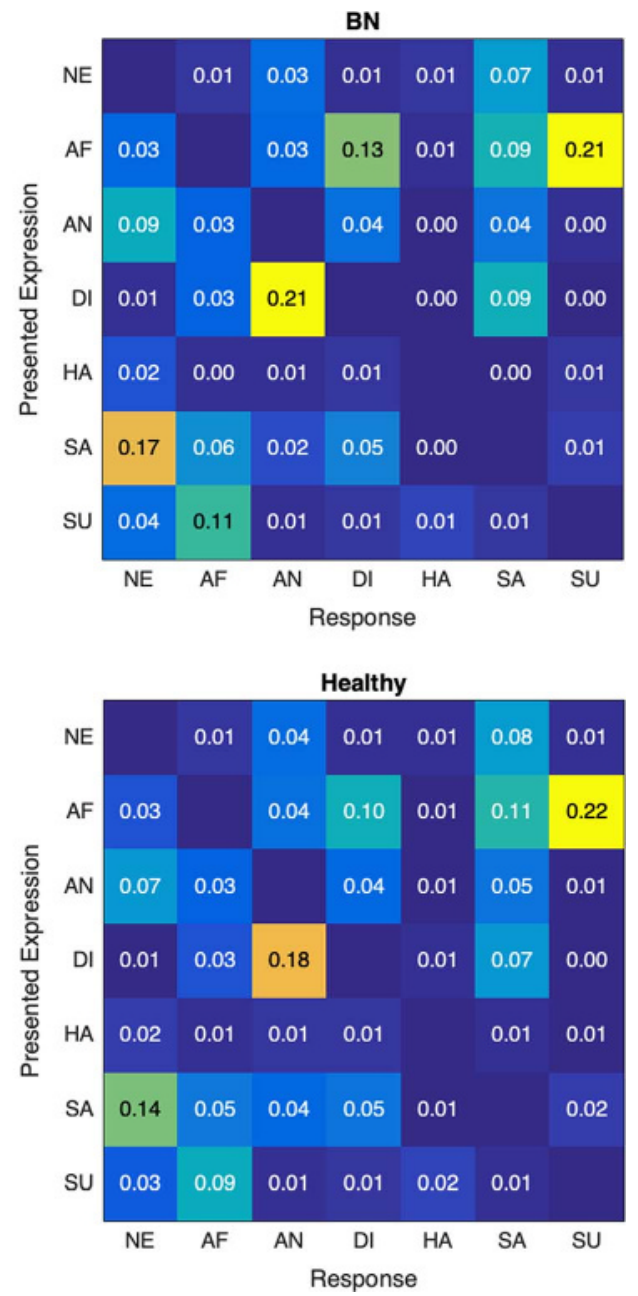

FIGURE 2. Confusion matrix: the group average response errors during the QUEST procedure for each facial expression of emotion $(N=308)$. Notes: $\mathrm{SU}=$ surprise, $\mathrm{SA}=$ sadness, $\mathrm{HA}=$ happiness, $\mathrm{DI}=$ disgust, $\mathrm{AN}=$ anger, $\mathrm{AF}=$ fear, $\mathrm{NE}=$ neutral. Each row displays one of the six presented facial expressions plus neutral, whereas each column shows the average confusion response given by the observers. The color map is correspondent to the number displayed in the matrix, with dark blue tones indicating low confusion, whereas yellow color indicates high confusion. Color image is available only in online version (www.psychosomaticmedicine.org). 
result was found for BMI increasing the threshold for the neutral expression $\left(R_{\text {Change }}^{2}=.022, F(1,305)=4.97, p=.009\right)$, all other intervening variables were not significant $\left(R_{\text {Change }}^{2} \leq .002\right.$, $F(1,305) \leq 1.92, p \geq .359)$.

\section{Response Biases for Emotion Expressions}

To explore response biases, confusion matrix for each emotion expression separately for each diagnostic group was calculated. As shown in Figure 2, fear was the most frequently confounded expression in all groups with a confusion rate approximately $50 \%$ for all groups, and the most commonly miscategorized as surprise $(21 \%-24 \%)$. Happiness was rarely confounded with a confusion rate of $3 \%$ to $7 \%$. The second most common confusion rates were between the expressions disgust and anger (17\%-22\%) and between sadness and neutral (14\%-18\%).

Moreover, as shown in Figure 3A, the similarity of confusion matrix among participants is rather high regardless of groups. The average between-subject correlation of response pattern is at 0.901 [0.734, 0.972] (bracket shows .025 and .975 quantiles of the distribution). We also compared the within-group correlation (orange dots and line in Figure 3B) with the between-group correlation (compare with HCs as baseline, blue dots and line in Figure 3B). No significances were found using the random sample permutation test comparing the distribution, indicating that the participants from all groups show a similar response profile.

\section{DISCUSSION}

The scope of the present study was to shed light on facial emotion recognition thresholds and biases in women experiencing $\mathrm{AN}$ and $\mathrm{BN}$ compared with healthy women and women experiencing mixed mental disorders (depressive and anxiety disorder). We assumed impairments in patients with $\mathrm{AN}$, whereas patients experiencing $\mathrm{BN}$ would perform similarly to HCs. The mixed mental disorder group was included as a clinical control group, because studies comparing EDs with other mental disorders are lacking. We additionally considered person-related factors (i.e., BMI, emotional clarity, severity of depressiveness, and ED pathology), which might influence the threshold of correct identification of facial emotion expressions and applied a new paradigm, which assesses an individual's emotion recognition threshold for six basic facial emotion expressions and a neutral expression.

Our findings in a large healthy and clinical sample, where in total just three persons were excluded from analyses (delivering only "don't know" answers), confirm the practicability of the paradigm presented in the initial study (5) in a clinical group. The paradigm established individual thresholds of facial emotion recognition, which were similar to the Rodger and colleagues (5) study. These findings confirm the reliability and validity of the paradigm.

With respect to our first study objective to detect thresholds of individual emotion recognition and related differences among groups, we found strong within-subject effects regarding particular emotions. As expected, facial expression of happiness was discriminated at the lowest threshold by all groups. Much stronger signals were required to identify correctly fear in facial expressions. In line with this, fear was the most commonly confounded expression in all groups. Most difficulties were shown in discriminating fear from surprise. This supports the finding of Rodger and colleagues (5), who reported that children at the age of 5 years showed the same emotion recognition thresholds as healthy adults for the emotions happiness and fear. In contrast to our expectations,
A

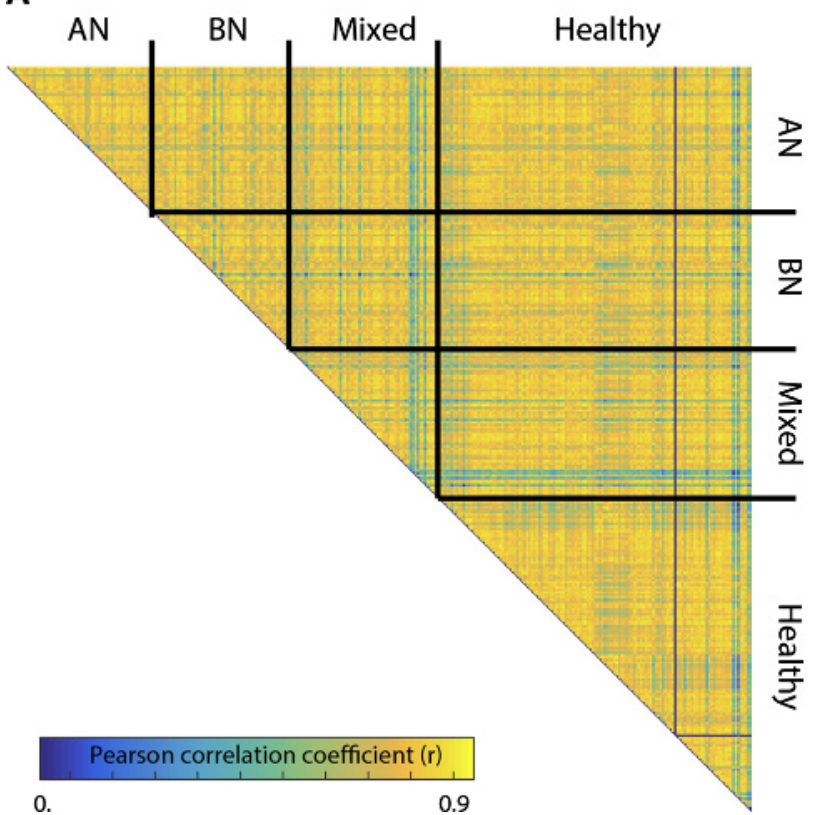

B

\section{Pearson correlation coefficient $(r)$}

FIGURE 3. Single-subject representational similarity matrix $(N=308)$. A, Correlation of the confusion matrix between each unique pair of participants, organized by different groups. As shown in the figure, participants make similar confusions (i.e., high correlation). B, Summary of each cell in the similarity matrix as shown in Figure 3A. The orange color dots show the similarity for each pair of participants within the same group, whereas blue color dots show the similarity for between the clinical group and HCs. Error bars show the interval where $95 \%$ of the data point falls in. Color image is available only in online version (www.psychosomaticmedicine.org). 
analyses in our sample only revealed marginal differences between groups. The hypothesis that women experiencing AN would show higher emotion recognition thresholds and higher confusion rates was not confirmed. According to contrast analyses, the only difference we found was for the mixed and the BN group, who needed higher signal strength to identify the emotion disgust than the $\mathrm{HC}$ and AN group. This result was supported by a relatively high confusion rate. It may be speculated that our mixed and $\mathrm{BN}$ group revealed more problems when recognizing an emotional stimulus of higher complexity or ambiguity. However, these findings are rather counterintuitive, because anxiety seems to be related with a disgust proneness and thus an accelerated processing of disgust related information, whereas in depression, lower biases to distinguish positive signals in ambiguous faces have been found $(17,58)$. In sum, our findings suggest that basic abilities to correctly identify facial emotion expressions do not differ between women experiencing $\mathrm{AN}, \mathrm{BN}$, and mixed mental disorders nor HCs. Only minimal effects were found for age in respect of the emotions anger and surprise and for BMI in respect of the neutral expression. Emotional clarity, the degree of depressiveness, and ED pathology did not alter the performance in the facial emotion recognition task, which is in line with the Brewer and colleagues (37) study but contradicts the results of Ridout and colleagues (33) and Sharpe and colleagues (59), who found lower correct identification of emotion expressions in individuals with higher ED pathology.

According to our second study aim, we investigated whether certain emotions were more frequently confused and whether confusion patterns differentiate between patients experiencing EDs and the $\mathrm{HC}$ as well as the mixed group. In general, the confusion rates in our sample correspond with the results of Rodger and colleagues (5) in a healthy population of different ages. In our sample, fear and surprise were most often confused. This finding is in line with literature, where this confusion is explained with their visual similarity based on shared muscles activities especially in the eye region (60). Relatively high confusion rates between sad and neutral faces could partly be explained by the findings of a recent meta-analysis in patients experiencing major depression. This revealed a bias toward sadness in that neutral, happy, or ambiguous facial emotion expressions were more confused with sadness (16). The authors explain these findings by an increased vigilance toward sadness cues, whereas there is evidence that general or emotion-specific recognition accuracy seems to be preserved.

In contrast to our expectations, we did not find specific or different confusion biases in any of our study groups. This result may partly be related to the neutral and rather technical presentation of the facial emotion expression stimuli in our study and the short presentation duration. Our stimuli neither were self-related nor have they been associated with a previous emotion induction. The emotion recognition task took place at the beginning of an experimental procedure, where participants were in a rather neutral mood. Thus, our findings are unlikely to be influenced by information related to the self or emotion induction before the test.

To summarize, in our large sample of women experiencing EDs, facial emotion recognition is preserved and confusion rates are comparable with the findings in the $\mathrm{HC}$ and the mixed mental disorder group. All participants seem to successfully draw their attention to emotional stimuli (facial expressions) and identify them correctly according to the Kret and Ploeger model (6). However, our study does not allow statements regarding nonfacial emotional stimuli such as recognizing emotions from voices, where other studies found impairments (for an overview, see, e.g., (19)). Future studies could apply a stepwise procedure with additional tasks to identify the step of emotion processing, where self-evaluation mechanisms or emotional valence influences emotion recognition thresholds and confusion rates in EDs compared with healthy or other clinical groups. Our study revealed that patients experiencing EDs are capable of recognizing basic emotion in the faces of others. This is not in accordance with the assumption made in the cognitive-interpersonal maintenance model of AN (18) assuming difficulties in emotion recognition and a bias toward negative emotions to be an important part that contributes to difficulties in social processing and emotion avoidance. However, our study does not allow statements regarding other emotion related processes that are relevant in social interaction, such as the ability to correctly interpret or express one's own emotions and intentions. Our findings further indicate that patients with EDs may rather fail in the interpretation process of emotions in complex scenarios including body movement and different tonality of voices or with respect to signaling of emotions in social interactions. The latter two processes are essential ingredients of social processing traits. In addition, especially AN patients might fail to functionally regulate the inner emotional response or state, which may contribute to emotional avoidance $(40,61)$.

Several limitations need to be considered when interpreting the results. First, groups are not age matched, nor was the HC group representative because it was composed of students, whereas patient samples were more heterogeneous. Second, further factors such as alexithymia known to influence emotion recognition (37) were not assessed in the present study. However, the inclusion of emotional clarity of the DERS subscale as a proxy thereof did not alter emotion recognition performance in our samples. Third, the AN group was composed of 30 patients $(49.2 \%)$ with the restrictive subtype and 28 patients (45.9\%) with the binge/purging subtype (for 3 patients [4.9\%], the subtype is unknown). Although previous studies refer to possible differences in emotion recognition between subtypes (e.g., 24), we did not run corresponding analysis because subsamples were too small.

Nevertheless, the present study has several strengths such as the large sample size and the inclusion of different clinical groups identified by clinical interviews (DIPS) as well as an HC group, which allowed to specify similarities and differences between the healthy and mentally ill state as well as between different disorder types. We applied the QUEST paradigm, which has shown to be a feasible, sensitive, and valid measure to identify basic competences in emotion recognition (5). Future studies should attempt to increase the ecological validity of the stimulus material and include dynamic facial expressions as well as bodily or acoustic cues not only in female but also in male participants (6). In addition, future paradigms should allow assessing not only basic information processing during emotion recognition such as in QUEST but also attentional processes related to the identification of emotional faces and subsequent attributional and interpretation mechanisms. These could provide additional information about the processing of emotional information (6).

To conclude, our findings indicate intact basic abilities in facial emotion recognition in EDs. Thus, explanations for difficulties in emotion processing in EDs could not be found at this early step of emotion processing and may rather relate to interpretation of recognized emotions under more complex circumstances. If our 
findings are replicated and new studies can identify concrete steps where ED-related specific deficits occur, it would be helpful to focus on the development of trainings for specific stages of emotion processing and regulation.

Source of Funding and Conflicts of Interest: This study was supported by grants from the Research Fund of the University of Fribourg, Switzerland (Grant Number 419), the Swiss Anorexia Nervosa Foundation (Grant Number 22-12), the Swiss National Science Foundation (Grant Number 100014L_149416/1), and the German Research Foundation (Grant Number SCHN 415/4-1). The authors report no conflicts of interest.

\section{REFERENCES}

1. Frith CD, Frith U. Social cognition in humans. Curr Biol 2007;17:R724-32.

2. Kilts CD, Egan G, Gideon DA, Ely TD, Hoffman JM. Dissociable neural pathways are involved in the recognition of emotion in static and dynamic facial expressions. Neuroimage 2003;18:156-68

3. Ekman P. Facial expressions of emotion: an old controversy and new findings. Philos Trans R Soc Lond B Biol Sci 1992;335:63-9.

4. Adolphs R. Recognizing emotion from facial expressions: psychological and neurological mechanisms. Behav Cogn Neurosci Rev 2002;1:21-62.

5. Rodger H, Vizioli L, Ouyang X, Caldara R. Mapping the development of facial expression recognition. Dev Sci 2015;18:926-39.

6. Kret ME, Ploeger A. Emotion processing deficits: a liability spectrum providing insight into comorbidity of mental disorders. Neurosci Biobehav Rev 2015;52: $153-71$.

7. Topper M, Emmelkamp PM, Watkins E, Ehring T. Prevention of anxiety disorders and depression by targeting excessive worry and rumination in adolescents an young adults: a randomized controlled trial. Behav Res Ther 2017;90:123-36.

8. Campbell-Sills L, Barlow DH, Brown TA, Hofmann SG. Effects of suppression and acceptance on emotional responses of individuals with anxiety and mood disorders. Behav Res Ther 2006;44:1251-63.

9. Daros AR, Zakzanis KK, Ruocco AC. Facial emotion recognition in borderline personality disorder. Psychol Med 2013;43:1953-63.

10. Domes G, Schulze L, Herpertz SC. Emotion recognition in borderline personality disorder — a review of the literature. J Pers Disord 2009;23:6-19.

11. Kohler CG, Walker JB, Martin EA, Healey KM, Moberg PJ. Facial emotion perception in schizophrenia: a meta-analytic review. Schizophr Bull 2010;36: 1009-19.

12. Uljarevic M, Hamilton A. Recognition of emotions in autism: a formal metaanalysis. J Autism Dev Disord 2013;43:1517-26.

13. Kohler CG, Hoffman LJ, Eastman LB, Healey K, Moberg PJ. Facial emotion perception in depression and bipolar disorder: a quantitative review. Psychiatry Res 2011;188:303-9.

14. Dalili MN, Penton-Voak IS, Harmer CJ, Munafo MR. Meta-analysis of emotion recognition deficits in major depressive disorder. Psychol Med 2015;45:1135-44.

15. Plana I, Lavoie MA, Battaglia M, Achim AM. A meta-analysis and scoping review of social cognition performance in social phobia, posttraumatic stress disorder and other anxiety disorders. J Anxiety Disord 2014:28:169-77.

16. Bourke C, Douglas K, Porter R. Processing of facial emotion expression in major depression: a review. Aust N Z J Psychiatry 2010;44:681-96.

17. Olatunji BO, Armstrong T, Elwood L. Is disgust proneness associated with anxiety and related disorders? A qualitative review and meta-analysis of group comparison and correlational studies. Perspect Psychol Sci 2017;12:613-48.

18. Treasure J, Schmidt U. The cognitive-interpersonal maintenance model of anorexia nervosa revisited: a summary of the evidence for cognitive, socio-emotional and interpersonal predisposing and perpetuating factors. J Eat Disord 2013;1:13

19. Caglar-Nazali HP, Corfield F, Cardi V, Ambwani S, Leppanen J, Olabintan O, Deriziotis S, Hadjimichalis A, Scognamiglio P, Eshkevari E, Micali N, Treasure J. A systematic review and meta-analysis of 'Systems for Social Processes' in eating disorders. Neurosci Biobehav Rev 2014;42:55-92.

20. Baron-Cohen S, Wheelwright S, Hill J, Raste Y, Plumb I. The "Reading the Mind in the Eyes" test revised version: a study with normal adults, and adults with Asperger syndrome or high-functioning autism. J Child Psychol Psychiatry 2001;42:241-51

21. Baron-Cohen S, Bowen DC, Holt RJ, Allison C, Auyeung B, Lombardo MV, Smith P, Lai MC. The "Reading the Mind in the Eyes" test: complete absence of typical sex difference in $\sim 400$ men and women with autism. PLoS One 2015 ; 10:e0136521.

22. Vellante M, Baron-Cohen S, Melis M, Marrone M, Petretto DR, Masala C, Preti A The "Reading the Mind in the Eyes" test: systematic review of psychometric properties and a validation study in Italy. Cogn Neuropsychiatry 2013;18:326-54.
23. Harrison A, Tchanturia K, Treasure J. Attentional bias, emotion recognition, and emotion regulation in anorexia: state or trait? Biol Psychiatry 2010;68:755-61.

24. Harrison A, Sullivan S, Tchanturia K, Treasure J. Emotional functioning in eating disorders: attentional bias, emotion recognition and emotion regulation. Psychol Med 2010;40:1887-97.

25. Harrison A, Sullivan S, Tchanturia K, Treasure J. Emotion recognition and regulation in anorexia nervosa. Clin Psychol Psychother 2009;16:348-56.

26. Oldershaw A. Emotional Theory of Mind in Anorexia Nervosa: State or Trait? [dissertation]. London, London: King's College London (University of London); 2009.

27. Kenyon M, Samarawickrema N, Dejong H, Van den Eynde F, Startup H, Lavender A, Goodman-Smith E, Schmidt U. Theory of mind in bulimia nervosa. Int J Eating Dis 2012;45:377-84.

28. Medina-Pradas C, Navarro JB, Álvarez-Moya EM, Grau A, Obiols JE. Emotional theory of mind in eating disorders. Int J Clin Health Psychol 2012;12:189.

29. Scrimin S, Moscardino U, Capello F, Altoe G, Axia G. Recognition of facial expressions of mixed emotions in school-age children exposed to terrorism. Dev Psychol 2009;45:1341-52.

30. Jansch C, Harmer C, Cooper MJ. Emotional processing in women with anorexia nervosa and in healthy volunteers. Eat Behav 2009;10:184-91

31. Cardi V, Corfield F, Leppanen J, Rhind C, Deriziotis S, Hadjimichalis A, Hibbs R, Micali N, Treasure J. Emotional processing, recognition, empathy and evoked facial expression in eating disorders: an experimental study to map deficits in social cognition. PLoS One. 2015;10:e133827.

32. Dapelo MM, Surguladze S, Morris R, Tchanturia K. Emotion recognition in blended facial expressions in women with anorexia nervosa. Eur Eat Disord Rev 2016;24: $34-42$.

33. Ridout N, Wallis DJ, Autwal Y, Sellis J. The influence of emotional intensity on facial emotion recognition in disordered eating. Appetite 2012;59:181-6.

34. Cardi V, Di Matteo R, Corfield F, Treasure J. Social reward and rejection sensitivity in eating disorders: an investigation of attentional bias and early experiences. World J Biol Psychiatry 2013;14:622-33.

35. Cserjési R, Vermeulen N, Lénárd L, Luminet O. Reduced capacity in automatic processing of facial expression in restrictive anorexia nervosa and obesity. Psychiatry Res 2011;188:253-7.

36. Dear BF, Sharpe L, Nicholas MK, Refshauge K. The psychometric properties of the dot-probe paradigm when used in pain-related attentional bias research. J Pain 2011;12:1247-54

37. Brewer R, Cook R, Cardi V, Treasure J, Bird G. Emotion recognition deficits in eating disorders are explained by co-occurring alexithymia. R Soc Open Sci 2015; 2:140382. Available at: https://doi.org/10.1098/rsos.140382. Accessed January 10, 2019.

38. Lule D, Schulze UM, Bauer K, Scholl F, Muller S, Fladung AK, Uttner I. Anorexia nervosa and its relation to depression, anxiety, alexithymia and emotional processing deficits. Eat Weight Disord 2014;19:209-16.

39. Oldershaw A, Hambrook D, Stahl D, Tchanturia K, Treasure J, Schmidt U. The socio-emotional processing stream in anorexia nervosa. Neurosci Biobehav Rev 2011;35:970-88.

40. Rawal A, Park RJ, Williams JM. Rumination, experiential avoidance, and dysfunctional thinking in eating disorders. Behav Res Ther 2010;48:851-9.

41. Watson AB, Pelli DG. QUEST: a Bayesian adaptive psychometric method. Percept Psychophys 1983;33:113-20.

42. Munsch S. Study protocol: psychological and physiological consequences of exposure to mass media in young women - an experimental cross-sectional and longitudinal study and the role of moderators. BMC psychology 2014;2:37.

43. APA. Diagnostic and Statistical Manual of Mental Disorders: DSM-IV-TR. 4th ed. Washington DC: American Psychiatric Association; 2000.

44. Friborg O, Martinussen M, Kaiser S, Øvergård KT, Martinsen EW, Schmierer P, Rosenvinge JH. Personality disorders in eating disorder not otherwise specified and binge eating disorder: a meta-analysis of comorbidity studies. J Nerv Ment Dis 2014;202:119-25.

45. Keski-Rahkonen A, Mustelin L. Epidemiology of eating disorders in Europe: prevalence, incidence, comorbidity, course, consequences, and risk factors. Curr Opin Psychiatry 2016;29:340-5.

46. Schneider S, Margraf J. DIPS: Diagnostisches Interview Bei Psychischen Störungen Handbuch-Interviewleitfaden-Protokollbogen. Berlin: Springer Verlag; 2011.

47. Rohde P, Lewinsohn PM, Seeley JR. Comparability of telephone and face-to-face interviews in assessing axis I and II disorders. Am J Psychiatry 1997;154:1593-8.

48. Beck AT, Steer RA, Brown GK. Beck Depression Inventory-Second Edition Manual. San Antonio, TX: The Psychological Corporation; 1996.

49. Hautzinger M, Keller F, Kuehner C. Beck Depressions-Inventar Revision (BDI-II). Manual. Frankfurt am Main: Pearson Assessment \& Information; 2009.

50. Hilbert A, Tuschen-Caffier B. Eating Disorder Examination-Questionnaire: Deutschsprachige Übersetzung. Verlag für Psychotherapie: Münster; 2006.

51. Fairburn CG, Cooper Z, Shafran R. Cognitive behaviour therapy for eating disorders: a "transdiagnostic" theory and treatment. Behav Res Ther 2003;41: 509-28.

52. Mond JM, Hay PJ, Rodgers B, Owen C, Beumont PJ. Validity of the Eating Disorder Examination Questionnaire (EDE-Q) in screening for eating disorders in community samples. Behav Res Ther 2004;42:551-67. 


\section{ORIGINAL ARTICLE}

53. Gratz KL, Roemer L. Multidimensional assessment of emotion regulation and dysregulation: development, factor structure, and initial validation of the difficulties in emotion regulation scale. J Psychopathol Behav 2004;26:41-54.

54. Ehring T, Fischer S, Schnulle J, Bosterling A, Tuschen-Caffier B. Characteristics of emotion regulation in recovered depressed versus never depressed individuals Pers Indiv Differ 2008;44:1574-84.

55. Batty M, Taylor MJ. Early processing of the six basic facial emotional expressions Brain Res Cogn Brain Res 2003;17:613-20.

56. Eimer M, Holmes A. An ERP study on the time course of emotional face processing. Neuroreport 2002;13:427-31.

57. Kriegeskorte N, Mur M, Bandettini P. Representational similarity analysis connecting the branches of systems neuroscience. Front Syst Neurosci 2008;2:-4
58. Sanchez A, Romero N, Maurage P, De Raedt R. Identification of emotions in mixed disgusted-happy faces as a function of depressive symptom severity. J Behav Ther Exp Psychiatry 2017;57:96-102

59. Sharpe E, Wallis DJ, Ridout N. The influence of variations in eating disorder-related symptoms on processing of emotional faces in a non-clinical female sample: an eyetracking study. Psychiatry Res 2016;240:321-7.

60. Chamberland J, Roy-Charland A, Perron M, Dickinson J. Distinction between fear and surprise: an interpretation-independent test of the perceptual-attentional limitation hypothesis. Soc Neurosci 2017;751-68.

61. Wildes JE, Ringham RM, Marcus MD. Emotion avoidance in patients with anorexia nervosa: initial test of a functional model. Int J Eat Disord 2010;43: $398-404$. 